\title{
Reliable and Scalable State Management Using Migration of State Information in Web Services
}

\author{
Jongbae Moon, Hyungil Park, and Myungho Kim \\ \#313, School of Computing, Soongsil University, Sangdo-5 Dong, Dongjak-Gu, Seoul, \\ 156-743, Korea \\ \{jbmoon, hgpark\}@ss.ssu.ac.kr, kmh@ssu.ac.kr
}

\begin{abstract}
The WS-Resource framework (WSRF) was proposed as a reengineering and evolution of OGSI to be compatible with the current Web services conventions and specifications. Although WSRF separates state management from the stateless web service and provides a mechanism for state management, it still has some limitations. The tight-coupling between web services and their resource factories restricts the scalability. When the repository of stateful resources fails, stateful web services can not work. In this paper, we propose a new state management framework which is called State Management Web Service Framework (SMWSF) and implemented on Microsoft .NET framework. SMWSF provides reliability, flexibility, scalability, and security. SMWSF provides migration of state information and the service requestor can control the location of the repository of stateful resource. We also implemented a prototype system and conducted comparison experiments to evaluate performance.
\end{abstract}

Keywords: state management, web service, WSRF, migration.

\section{Introduction}

Web services are "software systems designed to support interoperable machine-tomachine interaction over a network" [1]. Though there has been some success of Web Service in the industry, Web service has been regarded as stateless and non-transient [2]. Recently, most of the web services, however, are stateful; state information is kept and used during the execution of applications.

To manage sate information within Web services framework, the Open Grid Services Infrastructure (OGSI) [3] and Web Service Resource Framework (WSRF) [4] were proposed. Both OGSI and WSRF are concerned with how to manipulate stateful resources. OGSI extends the power of Web services framework significantly by integrating support for transient, stateful service instances with existing Web services technologies. The Globus Toolkit 3 (GT3) [5] is an implementation of the OGSI specification and has become a de facto standard for Grid middleware. GT3 uses Grid service factory to create multiple instances of the Grid service, and the Grid service instances are stateful. However, because GT3 uses the same container for grid services, the

* This work was supported by the Soongsil University Research Fund. 
service container, which is a Grid service factory, has to be restarted whenever a new service joins. This will affect all existing services in the same container.

WSRF was introduced as a refactoring and evolution of OGSI, and provides a generic, open framework for modeling and accessing stateful resources using Web services. WSRF uses different container to manage stateful resources and Web services; WSRF separates state management from the stateless web services. Therefore, there is no loss of state information and other service instances should continue to work although the execution of a service instance fails. WSRF, however, still has some limitations [2]. Each Web service accompanies a WS-Resource factory. The tight coupling between the web service and the resource factory leads to scalability problem. Moreover, WSRF does not provide the flexibility of choosing the location of the state repository. This may introduce security problems although the service itself is trusted by the requestor; the requestor and provider may have different security policies. Moreover, when the state repository fails, the stateful web service does not work.

In this paper, we propose a new state management framework, which is implemented on Microsoft .NET Web services, and implement a prototype system. The prototype system makes Web services and their state management loosely-coupled and Web services can use another state management service, which is in another service provider, to provide scalability. The state information can migrate to another repository to enhance reliability, security, and scalability while Web services are running. The migration of the state information also provides the flexibility of choosing the location of the state repository. To provide security for the state information, the state management stores the state information with an encryption key which is sent by the requestor. Moreover, whenever the state information migrates, it is transferred through a security channel.

The rest of this paper is organized as follows. Section 2 summarizes the existing researches regarding the state management in Web services. Section 3 proposes a system model. Section 4 describes how to implement a prototype system. Section 5 evaluates the performance of the proposed system by conducting comparison experiments, and Section 6 concludes the paper.

\section{Related Works}

Web services have a problem that it is difficult to maintain state because web services are built on top of the stateless HTTP protocol. While Web service implementations are typically stateless, their interfaces frequently provide a user with the ability to access and manipulate state. In [6], three different models to keep and manage state information are proposed. However, maintaining state information has restriction on scalability. Moreover, to provide security for the state, extra works are required.

OGSI enables access to stateful resources with the maintenance and retrieval of state information. OGSI introduces the resource factory to create multiple instances of the Grid service. Because OGSI uses the same container for Grid services, the service container has to be restarted whenever a new service joins.

WSRF provides standardized patterns and interfaces to manage state information. WSRF introduces the WS-Resource factory to create the instance of stateful resources. When a web service fails, WSRF can restore the state information by 
separating state management from a web service. WSRF, however, has some limitation to manage the state. The tight coupling between Web services and their factories leads to scalability problem.

In [2], a generic state management service, which separates state management from Web services, is introduced to overcome WSRF's limitations. Besides the reduction of work for service developers, scalability is enhanced by the flexible deployment of the state management service. However, once the state information is stored in a stateful resource, the requestor can not change the location of the repository. Therefore, although the Web service and state management service are trusted by the requestor, the repository may not guarantee security problems. Moreover, failure of the repository reduces the reliability and scalability.

\section{Proposed State Management Model}

In this section, we propose a new state management framework which overcomes the WSRF's limitation as mentioned above. The proposed framework is called the State Management Web Service Framework (SMWSF). SMWSF provides scalability, flexibility, security and reliability.

Fig. 1 shows the state management system model based on SMWSF. In this system model, state management, which is a Web service for creating and managing the instance of a stateful resource, is separated from web services. The service requestor can make web services use another state management service that exists in one of the other service providers. The state management service implements common interfaces to store the state information in some types of stateful resource. Therefore, service authors can easily develop web services regardless of the implementation of state management interfaces. Moreover, the state management service provides an interface that a stateful resource can migrate to another repository, as well as in another type. The state information is encrypted or decrypted before the state management service stores or restores it. Moreover, communications between the state management service and the stateful resource is established through a security channel.

A stateful resource is referred to as a WS-Resource in WSRF, and each WSResource is described by an XML document. In SMWSF, a stateful resource is implemented in some different types. The service requestor may want to change the location of the stateful resource because of security problems or failure of the repository. The state information can migrate to another repository chosen by the requestor, and the type of the stateful resource can be changed. In addition, migrating the stateful resource enhances reliability when the repository fails or does not work well. During the migration process, to guarantee security for the state information, communication is established through a security protocol, such as IPSec (IP Security).

Fig. 1 a) shows the process of using the Web service in SMWSF, and the details are described as follows. Fist of all, a service requestor sends a request including an encryption key to the service provider, choosing a type of the stateful resource and a location where the stateful resource is stored in. Then, the Web service generates an XML document including the state information, and sends a request with the generated XML document to the state management web service. After the state management service encrypts the state information with the requestor's encryption key, the 
encrypted state information is stored in a chosen place. Moreover as well as in a desired type. After that, the state management service returns the URI of the stateful resource to the Web service, and then the Web service returns the URI to the requestor. When the requestor requests the stateful resource to be moved, the Web service takes in the type and URI of the stateful resource from the requestor, and sends a migration request to the state management service. The state management service reads the corresponding resource, and then stores the state information in the specified location as well as specified type. In addition, Fig. 1 b) shows the details that the Web service changes the state management service with another one. The old state management service sends the URI of the stateful resource to the new state. After the new state management service gets in contact with the stateful resource, then the Web service communicate with the new state management service through a security channel.

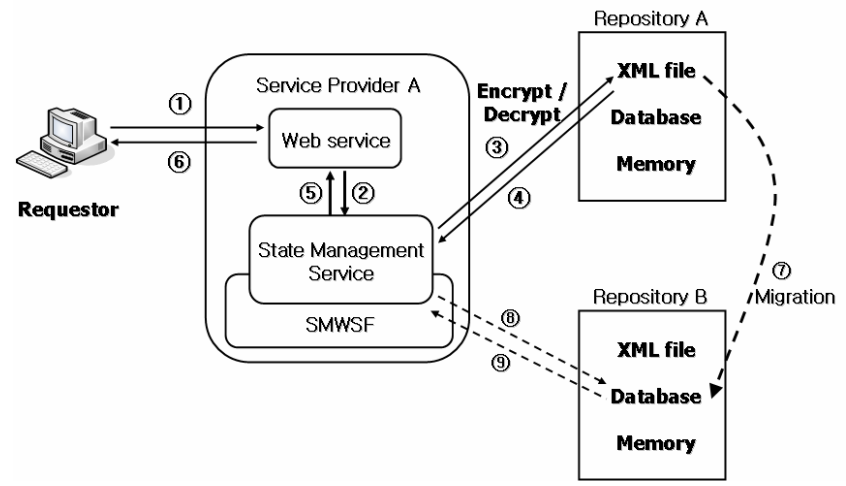

a) The state information can migrate from repository A to B.

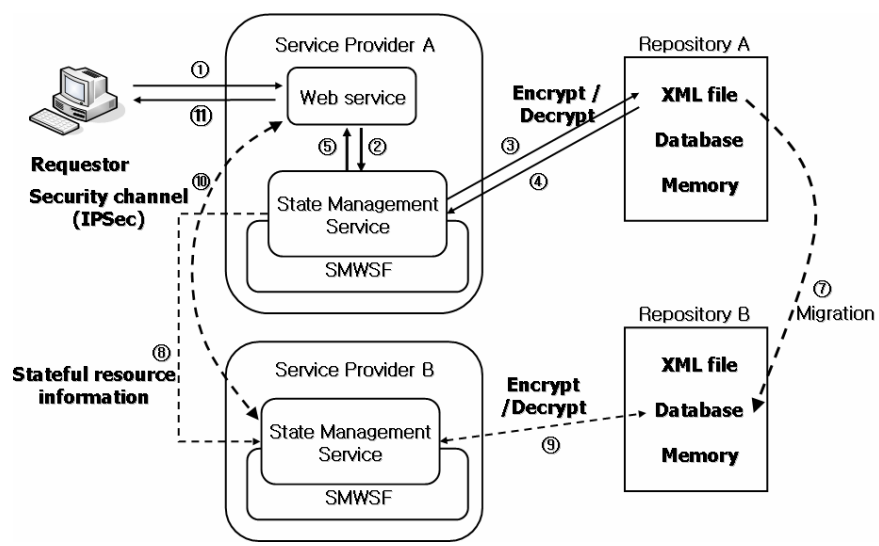

b) The service requestor can choose the state management service.

Fig. 1. Proposed State Management System Model 


\section{Implementation}

We implemented a prototype system to test SMWSF. We use ASP.NET in C\# to implement web service applications. Besides, we use Microsoft IIS (Internet Information Server) as a web application server. The prototype system is implemented on top of the Microsoft .NET framework. SMWSF implements basic WSRF specifications, such as WS-ResourceProperties [7] and WS-ResourceLifetime [8]. In the prototype system, a system is divided into three parts which are web services, a state management service including the proposed framework, and stateful resources. The system also provides libraries for implementing web services and lifetime management module for keeping track of the stateful resources created by the client requests.

In this system, every web service should be developed and implemented by importing libraries which is provided by SMWSF. Web services are ASP.NET web services. Service authors annotate their web service codes with metadata via .NET attributes. The port type attribute allows service authors to easily import the functionality that is defined by web service library into their service.

The web service libraries include state management interfaces, an XML generator, and a GUID generator. Web services use the state management interfaces, which are just defined but not implemented, to communicate with the state management service. The sate management web service is in charge of the implementation of the interfaces. Therefore, web services use another state management service which is provided by another service provider.

Web services generate service requestor's GUID (Global Unique ID) by using the GUID generator. The GUID, which is a 128bit integer number generated by using hash function, is used to grant access to a stateful resource. Web services generate an $\mathrm{XML}$ document to manipulate state information as a stateful resource by using the XML generator. In a web service, class-level data members are declared as part of the stateful resource via the [Resource] attribute to generate an XML document. The values of the class-level data members are saved into an XML document. The generated XML document includes a GUID and an encryption key in the <Header $>$ element. The class-level data member's name and value that are described in the [Resource] attribute in the web service are set in the $<$ Resource $>$ element. This XML document is encrypted with the encryption key, and then stored in the repository.

The state management service manages web services' state information. This service implemented as a web service. Service authors can make web services communicate with another state management web service provided by another service provider. The state management web service implements interfaces for state management, such as store, restore, deletion, and migration of stateful resources. To do this, the port type attribute is also used to import functionality that is defined in SMWSF. In the case of the migration, the state management web service generates an XML document from the repository first by using one of the stateful resource repository management modules. The XML document is stored in a desired place, as well as in a desired type. When the service requestor wants web services to use another state management service, the XML document is sent to the selected state management service, and then stored in the repository.

A stateful resource must persist between service invocations until the end of its lifetime, meaning that the state of a stateful resource after one invocation should be 
the same as its state before the next. Persistence can be achieved by holding the resource in memory, writing it to disk, or storing it in a database. The memory model provides the best response-time performance but least fault-tolerant. The file system model provides slow performance than other models, but provides the ability to survive server failure at the cost of some performance. The database model is slower than memory model, but provides scalability, fault-tolerance, and access to powerful query/discover mechanisms that are not present in the file system model. Therefore, in the proposed system the state resource repository management implements the stateful resource in these three types.

\section{Performance Evaluation}

In this section, we conducted two experiments for comparison to evaluate performance of SMWSF; we compared the system based on SMWSF with other systems implementing WSRF specification: GT4 and WSRF.NET. First, we implemented a calculator web service, and estimated the response time for creating, deleting, storing, restoring, and migrating state information of a web service. We performed each operation 1000 times, and then measured the average of the response time. The calculator service is a web service providing computation functionalities. Second, we implemented an airline booking web service on each framework. Then, we compared the service time, which is measured by the time in seconds for the client to receive the full response, as the number of clients increase. The airline booking service is a web service that retrieves airline schedules and then books seats. To perform this experiment automatically, we made the airline booking service book the first retrieved flight and reserve a fixed seat on the plane.

We used four identically configured machines that have an Intel Pentium $43.0 \mathrm{GHz}$ CPU with 1GB Ram and 80GB E-IDE 7200 RPM HDD. Two machines for SMWSF and one for WSRF.NET ran Windows 2003 Server Standard Edition. One for GT4 ran Linux Fedora Core 4 (Linux kernel 2.6.11). In SMWSF, stateful resources were implemented in three types, which were XML file, database, and memory; MySQL was used as a database for this experiment. GT4 stores state information in system memory. WSRF.NET implements WS-Resources using SQL Server 2000.

Table 1 shows the average response time for basic operations of the calculator service. All the numbers are in milliseconds. The Remote SMWSF is the case when the calculator service uses a remote state management service provided by another service provider. In this case, the average response time was slower than SMWSF and other systems because an additional communication cost between a web services and its state management service is needed. As compared with GT4 implementing stateful resource in memory model, SMWSF was faster in every test because GT4 is implemented in Java. As compared with WSRF.NET implementing stateful resource in database model, SMWSF had similar performance although there were additional overheads during encryption and decryption. Therefore, we could see SMWSF has as good performance as WSRF.NET has. 
Table 1. Average response time for basic operations

\begin{tabular}{|c|c|c|c|c|c|}
\hline \multicolumn{2}{|c|}{} & Create & Delete & Restore & Store \\
\hline \multicolumn{2}{|c|}{ GT4-Java } & $16.3 \mathrm{~ms}$ & $23.6 \mathrm{~ms}$ & $28.6 \mathrm{~ms}$ & $24.9 \mathrm{~ms}$ \\
\hline \multicolumn{2}{|c|}{ WSRF.NET } & $14.7 \mathrm{~ms}$ & $21.4 \mathrm{~ms}$ & $38.2 \mathrm{~ms}$ & $24.4 \mathrm{~ms}$ \\
\hline \multirow{3}{*}{ SMWSF } & File System & $15.3 \mathrm{~ms}$ & $23.5 \mathrm{~ms}$ & $32.8 \mathrm{~ms}$ & $22.3 \mathrm{~ms}$ \\
\cline { 2 - 5 } & Memory & $13.1 \mathrm{~ms}$ & $20.1 \mathrm{~ms}$ & $27.9 \mathrm{~ms}$ & $19.5 \mathrm{~ms}$ \\
\cline { 2 - 6 } & Database & $14.2 \mathrm{~ms}$ & $21.8 \mathrm{~ms}$ & $37.5 \mathrm{~ms}$ & $24.0 \mathrm{~ms}$ \\
\hline \multirow{2}{*}{$\begin{array}{c}\text { Remote } \\
\text { SMWSF }\end{array}$} & File System & $21.5 \mathrm{~ms}$ & $34.4 \mathrm{~ms}$ & $44.1 \mathrm{~ms}$ & $35.2 \mathrm{~ms}$ \\
\cline { 2 - 6 } & Memory & $19.4 \mathrm{~ms}$ & $30.8 \mathrm{~ms}$ & $37.4 \mathrm{~ms}$ & $30.4 \mathrm{~ms}$ \\
\cline { 2 - 6 } & Database & $20.8 \mathrm{~ms}$ & $32.9 \mathrm{~ms}$ & $47.4 \mathrm{~ms}$ & $36.4 \mathrm{~ms}$ \\
\hline
\end{tabular}

Fig. 2 shows the service time of the airline booking web service as the number of clients increase from 50 to 400. In this experiment, the Remote SMWSF is considered only if memory model. As the number of clients increase, the service time of GT4 was the fastest, followed by SMWSF, Remote SMWSF, and WSRF.NET. As might have been expected, the systems using memory model was faster than the systems using database and file system model. Moreover, Remote SMWSF was slower than SMWSF and GT4 because of additional communication cost and encryption overhead. GT4 had stable performance even though the number of clients increased because GT4 implements HTTP connection caching which reuses HTTP connection that was previously created. In the first experiment, the response time of SMWSF and WSRF.NET were comparable. In this experiment, as compared with WSRF.NET, SMWSF was faster because of the overhead caused by web service extension; WSRF.NET uses Microsoft Web Services Enhancement to provide SOAP message exchange. In addition, there were more SOAP data than SMWSF.

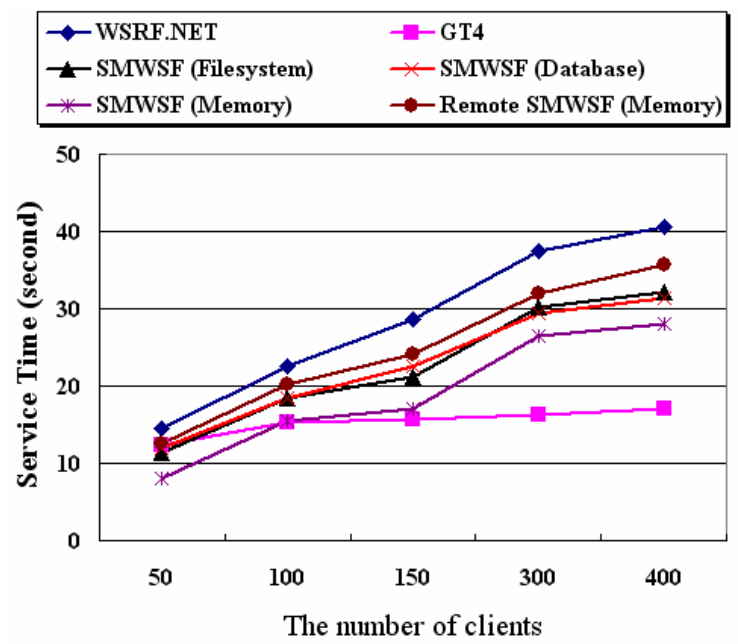

Fig. 2. Service time for an airline booking service according to the number of clients 


\section{Conclusions and Future Works}

In this paper, we proposed a new state management framework which provides scalability, flexibility, security, and reliability. This framework is called State Management Web Service Framework (SMWSF). We also implemented a prototype system that is based on the Microsoft .NET framework. In the prototype system, the state management is separated from web services. The loosely-coupled between the web service and the state management provides scalability. The flexibility is provided by making service requestors choose another state management service among the other service providers. The state information can migrate to another repository, where the type of stateful resource can be changed from one type to another. The migration of the state information enhances reliability and security when the repository fails or does not work well.

Many issues still remain to be addressed. Because of the loosely-coupled between the web service and the state management, some communication overhead is occurred. Moreover, the communication between sate management service and the sateful resource is done through a security channel. We need to study for reducing this additional communication overhead. We need to implement many other components for the framework, especially WS-Notification specifications. In addition, more experiments must be conducted on fault-tolerance to evaluate performance the proposed system.

\section{References}

1. David, Booth, Hugo, Haas, Francis, McCabe, Eric, Newcomer, Michael, Champion, Chris, Ferris, David, Orchard: Web Services Architecture - W3C Working Draft 8 August 2003. http://www.w3.org/TR/2003/WD-ws-arch-20030808/

2. Y., Xie, Y.M., Teo: State Management Issues and Grid Services. International Conference on Grid and Cooperative Computing. LNCS, Vol. 3251 (2004) 17-25

3. S., Tuecke, K., Czajkowski, I., Foster, J., Frey, S., Graham, C., Kesselman, P., Vanderbilt: Open Grid Service Infrastructure (OGSI). (2002)

4. Czajkowski, K., Ferguson, D., Foster, I., Frey, J., Graham, S., Sedukhin, I., Snelling, D., Tuecke, S., Vambenepe, W.: The WS-Resource Framework. http://www.globus.org/wsrf/ (2004)

5. Globus Toolkit version 3. http://www.globus.org/

6. Xiang, Song, Namgeun, Jeong, Phillip, W., Hutto, Umakishore, Ramachandran, James, M., Rehg: State Management in Web Services. IEEE International Workshop on FTDCS'04 (2004)

7. Graham, S., Czajkwski, K., Ferguson, D., Foster, I., Frey, J., Leymann, F., Maguire, T., Nagaratnam, N., Nally, M., Storey, T., Sedukhin, I., Snelling, D., Tuecke, S., Vambenepe, W., Weerawarana, S.: WS-ResourceProperties. http://www-106.ibm.com/developerworks/ library/ws-resource/ws-resourceproperties.pdf (2004)

8. Frey, J., Graham, S., Czajkowski, C., Ferguson, D., Foster, I., Leymann, F., Maguire, T., Nagaratnam, N., Nally, M., Storey, T., Sedukhin, I., Snelling, D., Tuecke, S., Vambenepe, W., Weerawarana, S.: WS-ResourceLifetime. http://www-106.ibm.com/developerworks/ library/ws-resource/ws-resourcelifetime.pdf (2004) 